\title{
Nanometer germanium photodetector with aluminum surface plasmon antenna for enhanced photo-response
}

\author{
Fang-Fang REN*, Kah-Wee ANG ${ }^{\dagger}$, Guo-Qiang LO ${ }^{\ddagger}$, Dim-Lee KWONG \\ Institute of Microelectronics, A STAR (Agency of Science, Technology and Research), Singapore \\ 117685
}

\begin{abstract}
We present theoretical design process for plasmon-enhanced photodetectors with nanometer-scale germanium area. The nontraditional plasmonic metal aluminum is employed as the material of surface plasmon antenna instead of noble metals owing to its integration compatibility with existing silicon complementary metal-oxide-semiconductor technology. The electrode/antenna is patterned with shallow concentric grating surrounding a subwavelength aperture (bull's eye structure) for concentrating and guiding strong optical intensity into an ultra-small active area. The physical modeling and geometric parameters optimization are performed based on the finite-difference time-domain method. Due to the excitation of fundamental or 2nd-order Bloch surface plasmon polaritons, high absorption can be obtained at nearinfrared wavelengths of 1310 and $800 \mathrm{~nm}$.
\end{abstract}

Keywords: surface plasmon polaritons, bull's eye structure, photodetectors, FDTD

\section{INTRODUCTION}

Germanium (Ge) photodetectors have been actively pursued for optical interconnection/communication applications owing to its large absorption coefficient ${ }^{1}$ and its integration compatibility with silicon ( $\mathrm{Si}$ ) complementary metal-oxidesemiconductor (CMOS) process technology. In particular, the metal-semiconductor-metal (MSM) configured photodiodes have been widely used due to its relative ease of fabrication and high speed performance ${ }^{2,3}$. The photodetector can be made smaller by using a nanometer-scale active region; however, there is an inherent trade-off between speed and quantum efficiency in a MSM photodetector. As well known, the total $-3 \mathrm{~dB}$ bandwidth is defined by both the carrier transit and the resistance-capacitance $(R C)$ delay. The former requires a thinner depletion layer, while it in turn results in a large depletion layer capacitance. To suppress the increase of the capacitance, the active area of the diode should be decreased. Meanwhile, the smaller active area leads to lower photo-response. It is required that the overall performance could be boosted by strongly confining incident light within a small active area.

The optical near-field localized around the nanometer element supplies a potential approach to overcome this trade-off. However, the poor throughput from far field to near field prevents the advancement of practical devices. It is a promising method to make use of surface plasmon (SP) antennas to convert input light into surface plasmon polaritons (SPPs) and induce extremely strong optical intensity inside a small region. SPPs are modes of eigenvalue solutions of Maxwell's equations. They have nonzero wave vector in the transverse direction, which implies that they propagate along the metal-dielectric interface. One distinct feature of SPP modes is that they cannot be excited directly by incident light on a flat interface. Periodically structured metallic media are thus playing a key role because they can dramatically change the dispersion relation of the SPPs.

*e-mail: renff@ime.a-star.edu.sg, †e-mail: angkw@ime.a-star.edu.sg, †e-mail: logq@ime.a-star.edu.sg; phone: +65 6770 5739, fax: +65 67731914

Silicon Photonics and Photonic Integrated Circuits II, edited by Giancarlo Cesare Righini, Proc. of SPIE Vol. 7719, 77191U · (C) 2010 SPIE · CCC code: 0277-786X/10/\$18 · doi: 10.1117/12.854179 
To carry out this technology into semiconductors, in this paper, we design a plasmon-enhanced Ge MSM photodetector with bull's eye antenna for near-infrared wavelength applications. The bull's eye structure, which consists of periodic surface corrugation and a subwavelength aperture, is employed as SP antenna for efficient optical-to-electrical encoding in a nanometer-scale active volume. The underlying principle is that the extraordinary transmission through a subwavelength aperture can be dramatically increased via the excitation of Bloch SPP waves (fundamental or highorder) at the interface between air and bull's eye antenna due to grating coupling ${ }^{4-6}$.

\section{SP ANTENNA DESIGN AND OPTIMIZATION}

\subsection{Plasmonic metal}

Gold $(\mathrm{Au})$ and silver $(\mathrm{Ag})$ are the most commonly used plasmonic metals, but neither of them is compatible with current Si CMOS process. We hereby consider nontraditional plasmonic metal aluminum (Al) as the substitute for our bull's eye antennas due to its Si-compatibility. The permittivity values of metal Al, including the real part $\varepsilon_{1}$ (Figure 1 left) and imaginary part $\varepsilon_{2}$ (Figure 1 right), are shown in Figure 1 ranging from $340 \mathrm{~nm}$ to $1550 \mathrm{~nm}^{7}$. We performed the physical modeling based on such frequency dependent dielectric constants.
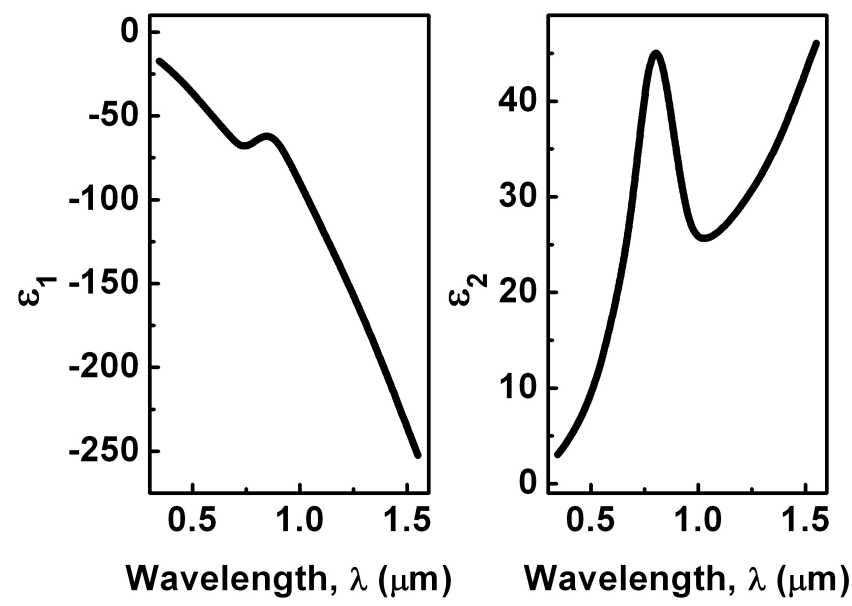

Figure 1. Frequency dependent dielectric constants of metal Al. Left is the real part. Right is the imaginary part.

\subsection{Bull's eye structure}

(a)

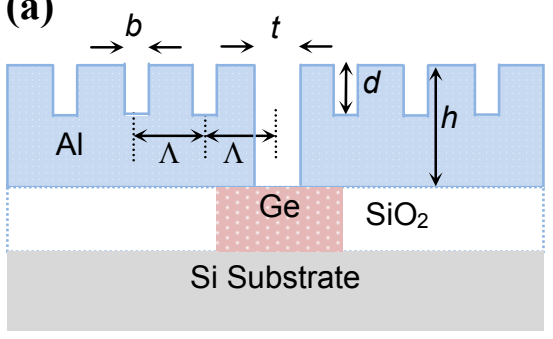

(b)

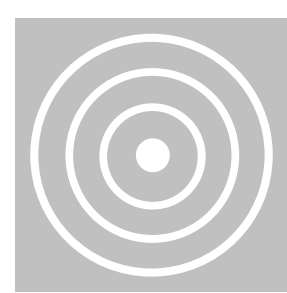

Figure 2. (a) The cross-sectional view of the Ge MSM photodetector with Al bull's eye antenna. (b) The top-view schematic of a bull's eye structure.

Figure 2 describes the cross-sectional view of our plasmonic photodetectors and the top-view schematic of a bull's eye structure. For vertical illumination, we investigate the optical near-field coupling effect of Al-based MSM electrodes patterned with bull's eye structure as a SP antenna. Structural parameters, including period length $\Lambda$, channel width $b$ (or wedge width $\Lambda-b$ ), channel depth $d$, diameter of aperture $t$, metal thickness $h$ and period number on each side of the 
aperture $P N$, will basically determine the SPP frequencies. Close to such resonances, a large enhancement of the optical near-field intensity will be induced on the exit side of the aperture, and thus enhance the photon-generated carriers within the ultra-small Ge mesa structure which is depleted under the reverse-bias condition.

\subsection{Grating period}

Among the design parameters, period length $\Lambda$ has the most significant influence on this issue. As shown in Figure 2a, the period length is defined as the distance between the centres of two nearest grating channels, and also from the centre of aperture to the centre of first channel. The value of $\Lambda$ can be roughly estimated according to the relation :

$$
\pm k_{S P P}=\frac{2 \pi}{\lambda} \sin \theta+m G
$$

Where $m$ is an integer, and $G=2 \pi / \Lambda, \lambda$ is the wavelength of incoming light in vacuum, $\theta_{\mathrm{i}}$ is the angle of incidence, and $k_{S P P}$ is the SPP propagation constant. The two resonant frequencies of the first order $(|m|=1)$ are fundamental Bloch SPP modes, and higher-order Bloch modes are corresponding to $|m|>1$. Under normal illumination, the two eigenmodes of $|m|$ order are degenerate, and the condition (1) can be simplified as

$$
\pm k_{S P P}=m G
$$

Based on the permittivity values of Al given in Figure 1, the solutions to Equation (2) are partly shown in Figure 3 for $m$ $=1,2$ and 3 as a function of the grating period $\Lambda$.

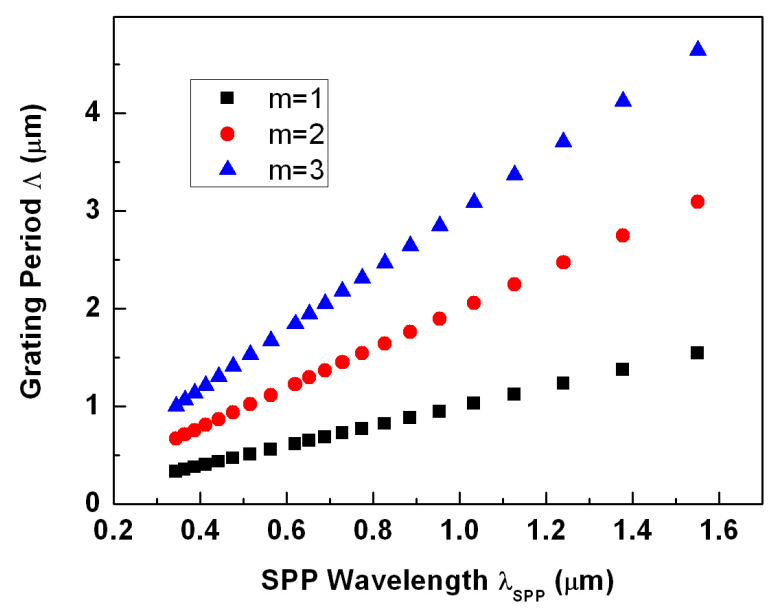

Figure 3. Estimate of the Bloch SPP wavelength for an air-Al grating interface. The results for the first three resonant orders are shown. The frequency dependent permittivity values of Al are given in Figure 1.

For a SPP wavelength of interest, such as 1310 or $800 \mathrm{~nm}$ in this work, the optimization of design parameters can be carried out by simulating the transmission spectra for high transmittance. For simplification, here we only discuss the design and optimization for $1310 \mathrm{~nm}$ in detail as an illustration. With choice of parameters as $\Lambda=1.23 \mu \mathrm{m}, b=0.2 \mu \mathrm{m}, d$ $=0.1 \mu \mathrm{m}, h=0.4 \mu \mathrm{m}, t=0.55 \mu \mathrm{m}$ and $P N=8$, the wavelength of $1310 \mathrm{~nm}$ can be tuned to the sharp transmission peak as shown in Figure 4, which is corresponding to the fundamental Bloch SPP mode. In order to obtain maximum absorption in the Ge region, we perform the parameter scan for channel depth and width, aperture diameter, as well as period number.

\subsection{Channel depth}

When examining the dependence of the transmission of the bull's eye antenna on the channel depth $d$, we fix $\Lambda=1.23$ $\mu \mathrm{m}, b=0.2 \mu \mathrm{m}, h=0.4 \mu \mathrm{m}, t=0.55 \mu \mathrm{m}$, and $P N=8$. Figure 5 shows the absorption enhancement versus the channel 
depth $d$ at wavelength of $1310 \mathrm{~nm}$. Here, we defined absorption power by volume integration of the light power entering into the Ge region, normalized by the incident wave flux. The absorption enhancement measures the enhancement factor of the light absorption per unit of the volume of the detector material. This factor is proportional to the improvement of the signal/noise ratio, because the thermal noise is mainly determined by the volume of the active detector material. It is found that the value of $d=0.1 \mu \mathrm{m}$ corresponds to the maximum in absorption enhancement, indicating the best choice of channel depth.

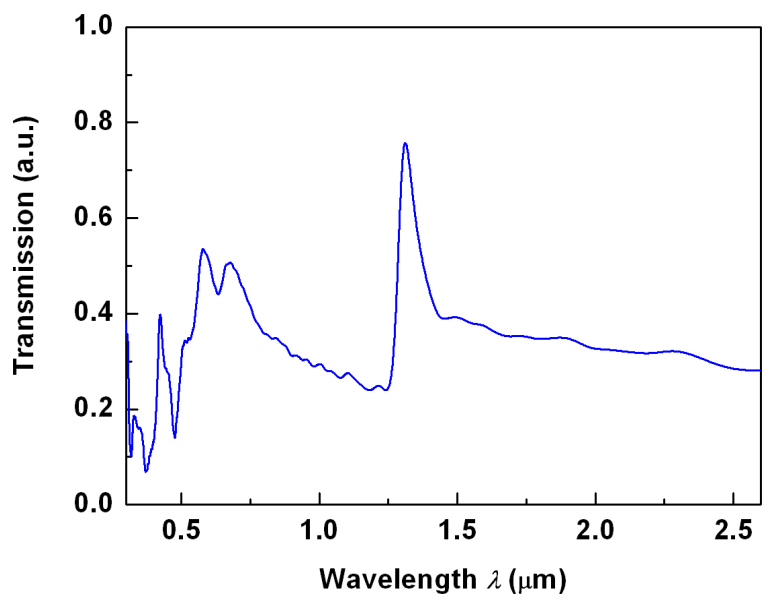

Figure 4. Plot of transmission versus wavelength for Al bull's eye structure with $\Lambda=1.23 \mu \mathrm{m}, b=0.2 \mu \mathrm{m}, d=0.1 \mu \mathrm{m}$, $h=0.4 \mu \mathrm{m}, t=0.55 \mu \mathrm{m}$ and $P N=8$.

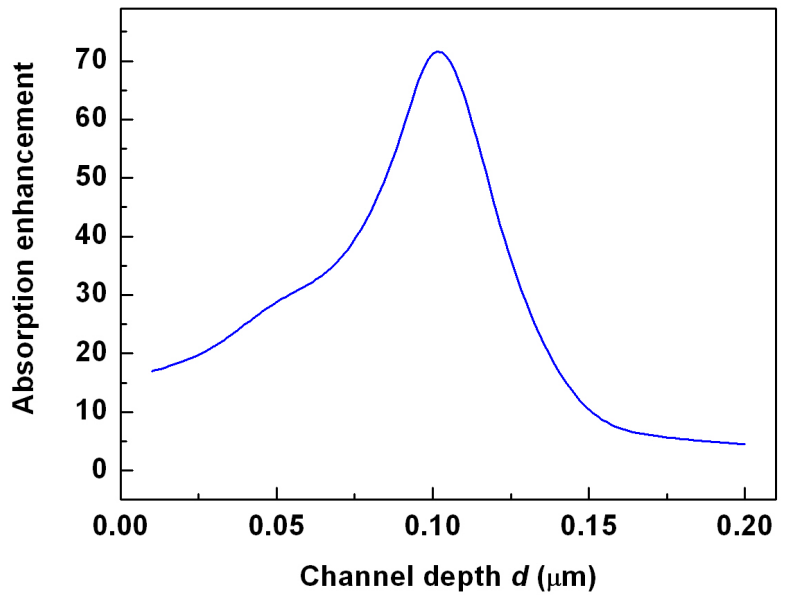

Figure 5. Plot of absorption enhancement versus channel depth $d$ for Al bull's eye structure with $\Lambda=1.23 \mu \mathrm{m}, b=0.2$ $\mu \mathrm{m}, h=0.4 \mu \mathrm{m}, t=0.55 \mu \mathrm{m}$ and $P N=8$. The illumination wavelength is $1310 \mathrm{~nm}$.

\subsection{Channel width}

By fixing $\Lambda=1.23 \mu \mathrm{m}, h=0.4 \mu \mathrm{m}, t=0.55 \mu \mathrm{m}$ and $P N=8$, as well as $d=0.1 \mu \mathrm{m}$ resulted from Section 2.4, we perform the scan for examining value $b$ from 0.02 to $0.8 \mu \mathrm{m}$ as shown in Figure 6 . A high-transmittance flat is found from $b=0.2$ to $0.6 \mu \mathrm{m}$ with a maximum appearing at $0.6 \mu \mathrm{m}$. It indicates that the SPP coupling is significant for a wide range of channel width. 


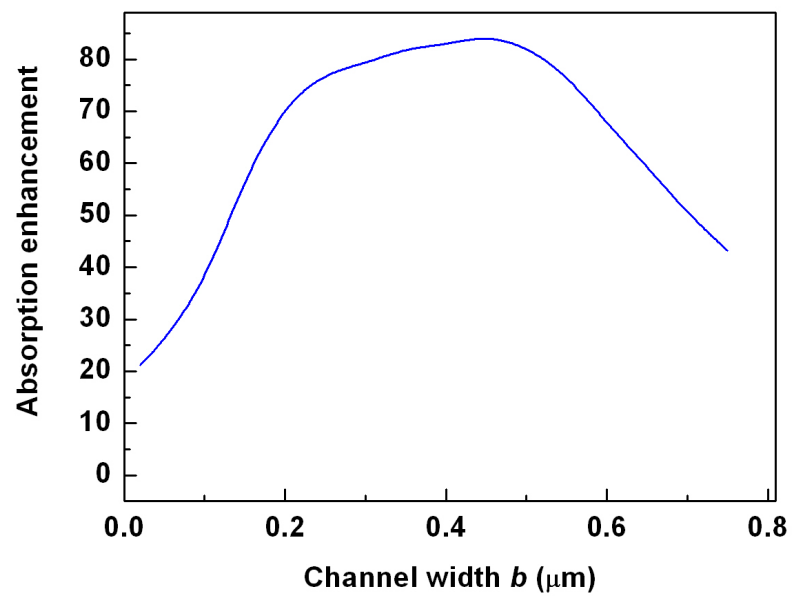

Figure 6. Plot of absorption enhancement versus channel width $b$ for Al bull's eye structure with $\Lambda=1.23 \mu \mathrm{m}, d=0.1$ $\mu \mathrm{m}, h=0.4 \mu \mathrm{m}, t=0.55 \mu \mathrm{m}$ and $P N=8$. The illumination wavelength is $1310 \mathrm{~nm}$.

\subsection{Aperture diameter}

By fixing $\Lambda=1.23 \mu \mathrm{m}, h=0.4 \mu \mathrm{m}, P N=8, d=0.1 \mu \mathrm{m}$ resulted from Section 2.4 and $b=0.45 \mu \mathrm{m}$ from Section 2.5, we can optimize the centre aperture for maximum absorption power or enhancement.
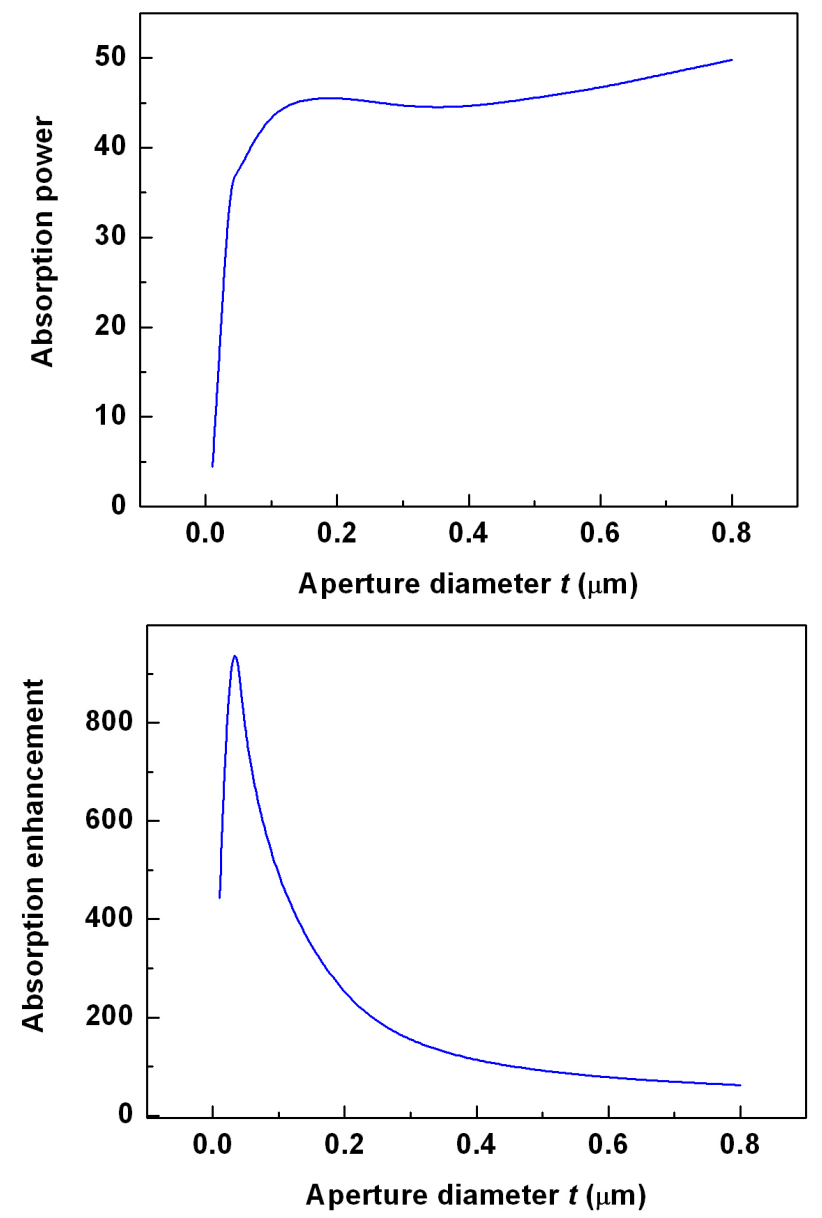

Proc. of SPIE Vol. 7719 77191U-5 
Figure 7. Plot of absorption power (top)/enhancement factor (bottom) versus aperture diameter $t$ for Al bull's eye structure with $\Lambda=1.23 \mu \mathrm{m}, d=0.1 \mu \mathrm{m}, b=0.45 \mu \mathrm{m}, h=0.4 \mu \mathrm{m}$ and $P N=8$. The illumination wavelength is $1310 \mathrm{~nm}$.

Figure 7 shows the dependence of absorption power (top) or enhancement factor (bottom) on the aperture diameter at wavelength of $1310 \mathrm{~nm}$. The absorption power represents the absolute transmission, which increases with the aperture diameter increasing as a main trend, partly due to the area increase of the aperture. The absorption enhancement shown in the bottom of Figure 7 is related to the normalized or relative transmission. We can find a factor of $\sim 950$ when aperture diameter $t$ is around $30 \mathrm{~nm}$. At this point, the ratio of the absorption power to the aperture size reaches maximum. The SPP coupling effect becomes weaker for larger $t$ beyond $30 \mathrm{~nm}$. Although the relative enhancement is reported in most of the published works for the consideration of physical significance, the absolute transmission is more important in practical applications. As seen in the top of Figure 7, the transmittance at $t=30 \mathrm{~nm}$ is not high enough for device application. For a fixed absorbing volume, the aperture should be chosen with sufficient exposed area, which will result more absorption power confined in the active region, leading to higher photocurrent.

\subsection{Period number}

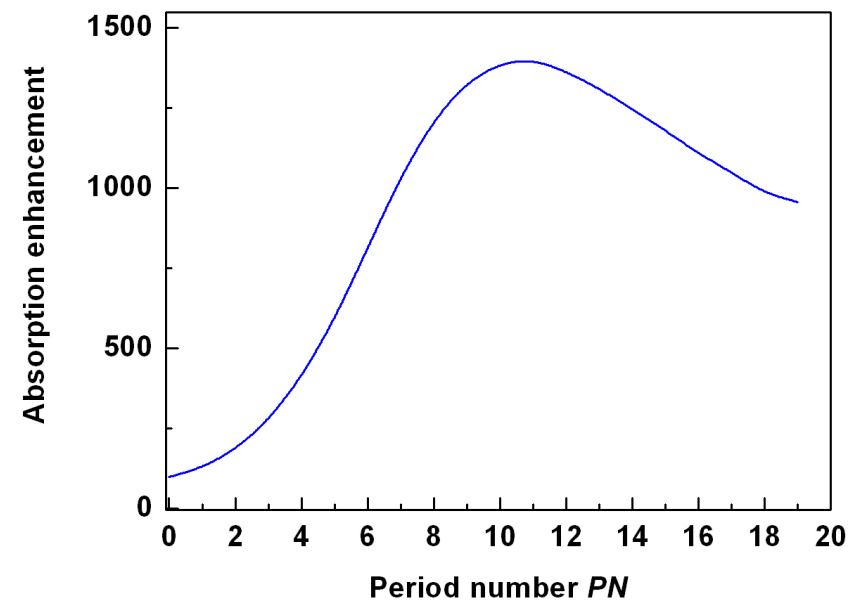

Figure 8. Plot of absorption enhancement factor versus period number $P N$ for Al bull's eye structure with $\Lambda=1.23 \mu \mathrm{m}$, $d=0.1 \mu \mathrm{m}, b=0.45 \mu \mathrm{m}, t=0.03 \mu \mathrm{m}$ and $h=0.4 \mu \mathrm{m}$. The illumination wavelength is $1310 \mathrm{~nm}$.

Figure 8 plots the dependence of absorption enhancement factor on the grating period number $P N$ on each side of the centre aperture. The other structural parameters are set as $\Lambda=1.23 \mu \mathrm{m}, d=0.1 \mu \mathrm{m}, b=0.45 \mu \mathrm{m}, t=0.03 \mu \mathrm{m}$ and $h=0.4$ $\mu \mathrm{m}$. As the number $P N$ increases, the absorption enhancement of the detector increases because the grating collects the additional optically excited surface waves ${ }^{9}$. The curve reaches a saturation point at $P N=10$ when the additional collection is balanced by re-radiation ${ }^{9}$. After that, the enhancement factor drops down slowly.

\subsection{High-order Bloch SPP modes}

For SPP wavelength shorter than $1 \mu \mathrm{m}$, ultra-small-size lithography might be critically required corresponding to the fundamental modes in terms of wavelength. Alternatively, we can design the period configuration with regard to higherorder modes for the advantage of longer period length ${ }^{10}$. In this work, a bull's eye structure with $\Lambda=1.5 \mu \mathrm{m}$ is designed and optimized for wavelength of $800 \mathrm{~nm}$ based on the excitation of 2nd-order Bloch SPP mode. The design process is similar with the wavelength of $1310 \mathrm{~nm}$ from Section 2.3 to 2.7 . 


\section{RESULTS AND DISCUSSION}

Table 1. Structural parameters of an optimized bull's eye antenna for high signal/noise ratio.

\begin{tabular}{|l|l|l|l|l|l|}
\hline SPP wavelength & $\begin{array}{l}\text { Period } \\
\text { length }\end{array}$ & $\begin{array}{l}\text { Channel } \\
\text { depth }\end{array}$ & $\begin{array}{l}\text { Channel } \\
\text { width }\end{array}$ & $\begin{array}{l}\text { Aperture } \\
\text { diameter }\end{array}$ & $\begin{array}{l}\text { Period } \\
\text { number }\end{array}$ \\
\hline $1310 \mathrm{~nm}$ (1st-order) & $1.23 \mu \mathrm{m}$ & $100 \mathrm{~nm}$ & $450 \mathrm{~nm}$ & $30 \mathrm{~nm}$ & 10 \\
\hline $800 \mathrm{~nm}$ (2nd-order) & $1.50 \mu \mathrm{m}$ & $70 \mathrm{~nm}$ & $250 \mathrm{~nm}$ & $10 \mathrm{~nm}$ & 10 \\
\hline
\end{tabular}

Table 1 listed all the optimized structural parameters of a bull's eye antenna, including period length $\Lambda$, channel depth $d$ and width $b$, aperture diameter $t$ and period number $P N$, for fundamental/2nd-order SPP excitation at 1310/800 $\mathrm{nm}$. During the numerical simulation, we simplified the three-dimension (3D) model to a 2D linear grating with a centre slit to reduce the computation time due to the cylindrical symmetry of a bull's eye structure. All the values listed in Table 1, including the ultra-small aperture diameter $(30$ or $10 \mathrm{~nm}$ ), are chosen to achieve strong high signal/noise ratio. For the aim to improve photo-response from a nanometer-scale Ge area with a fixed area, we can scale up the aperture size as discussed in Section 2.6 for the more effective detection and also the feasibility in IC fabrication laboratories.

\section{CONCLUSIONS}

We theoretically investigated the Al bull's eye antennas for plasmonic Ge MSM photodetectors at operation wavelength of 1310 and $800 \mathrm{~nm}$. Due to the excitation of fundamental or 2nd-order Bloch SPP waves, strong optical energy confinement can be realized in the Ge region directly below the centre aperture, leading to large quantum efficiency. Full-field simulation based on FDTD method was employed to design the basic structural parameters of the concentric grating and the centre subwavelength/deep subwavelength aperture. With the aim to achieve high absorption power/enhancement factor, we can obtain optimized structure dimensions of the antennas.

\section{REFERENCES}

1. Soref, R. A., "Silicon-based optoelectronics," Proc. IEEE, 81, 1687-1706 (1993).

2. Oh, J., Csutak, S. and Campbell, J. C., "High-speed interdigitated Ge PIN photodetectors," IEEE Photon. Technol. Lett., 14, 369-371 (2002).

3. Ang, K. W., Zhu, S., Yu, M., Lo, G. Q. and Kwong, D. L., "High-performance waveguided Ge-on-SOI metalsemiconductor-metal photodetectors with novel silicon-carbon (Si:C) Schottky barrier enhancement layer," IEEE Photon. Technol. Lett., 20, 754-756 (2008).

4. Ebbesen, T.W.; Lezec, H. J.; Ghaemi, H. F.; Thio, T. and Wolff, P. A., "Extraordinary optical transmission through sub-wavelength hole arrays," Nature, 391, 667-669 (1998).

5. Thio, T.; Pellerin, K. M.; Linke, R. A.; Lezec, H. J.and Ebbesen, T. W., "Enhanced light transmission through a single subwavelength aperture," Opt. Lett., 26, 1972-1974 (2001).

6. Degiron, A. and Ebbesen, T. W, "Analysis of the transmission process through single apertures surrounded by periodic corrugations,” Opt. Express, 12, 3694-3700 (2004).

7. Weaver, J. H. and Frederikse, H. P. R., [CRC handbook of Chemistry and Physics], CRC Press, Boca Raton, 12-133 (2001).

8. Barnes, W. L., Murray, W. A, Dintinger, J., Devaux, E. and Ebbesen, T. W., "Surface plasmon polaritons and their role in the enhanced transmission of light through periodic arrays of sub-wavelength holes in a metal film," Phys. Rev. Lett., 92, 107401 (2004).

9. Bhat, R. D. R., Panoiu, N. C., Brueck, S. R. J. and Osgood, Jr., R. M., "Enhancing the signal-to-noise ratio of an infrared photodetector with a circular metal grating," Opt. Express 16, 4588-4596 (2008). 
10. Kobyakov, A, Mafi, A., Zakharian, A. R., Darmanyan, S. A. and Sparks, K. B., "Fundamental and higher-order Bloch surface plasmons in planar bimetallic gratings on silicon and glass substrates," J. Opt. Soc. Am. B, 25, 1414-1421 (2008).

\section{REFERENCE LINKING}

Book: [7] Weaver, J. H. and Frederikse, H. P. R., [CRC handbook of Chemistry and Physics], CRC Press, Boca Raton, 12-133 (2001).

Journal Paper: [2] Oh, J., Csutak, S. and Campbell, J. C., "High-speed interdigitated Ge PIN photodetectors," IEEE Photon. Technol. Lett., 14, 369-371 (2002).

[3] Ang, K. W., Zhu, S., Yu, M., Lo, G. Q. and Kwong, D. L., "High-performance waveguided Ge-on-SOI metal-semiconductor-metal photodetectors with novel silicon-carbon (Si:C) Schottky barrier enhancement layer," IEEE Photon. Technol. Lett., 20, 754-756 (2008).

[4] Ebbesen, T.W.; Lezec, H. J.; Ghaemi, H. F.; Thio, T. and Wolff, P. A., "Extraordinary optical transmission through sub-wavelength hole arrays," Nature, 391, 667-669 (1998).

[5] Thio, T.; Pellerin, K. M.; Linke, R. A.; Lezec, H. J.and Ebbesen, T. W., "Enhanced light transmission through a single subwavelength aperture," Opt. Lett., 26, 1972-1974 (2001).

[6] Degiron, A. and Ebbesen, T. W, "Analysis of the transmission process through single apertures surrounded by periodic corrugations," Opt. Express, 12, 3694-3700 (2004).

[8] Barnes, W. L., Murray, W. A, Dintinger, J., Devaux, E. and Ebbesen, T. W., "Surface plasmon polaritons and their role in the enhanced transmission of light through periodic arrays of sub-wavelength holes in a metal film," Phys. Rev. Lett., 92, 107401 (2004).

[9] Bhat, R. D. R., Panoiu, N. C., Brueck, S. R. J. and Osgood, Jr., R. M., "Enhancing the signal-to-noise ratio of an infrared photodetector with a circular metal grating," Opt. Express 16, 4588-4596 (2008).

[10] Kobyakov, A, Mafi, A., Zakharian, A. R., Darmanyan, S. A. and Sparks, K. B., "Fundamental and higher-order Bloch surface plasmons in planar bimetallic gratings on silicon and glass substrates," J. Opt. Soc. Am. B, 25, 1414-1421 (2008).

Proceedings Paper: [1] Soref, R. A., "Silicon-based optoelectronics," Proc. IEEE, 81, 1687-1706 (1993). 